\title{
Avant que / avant de, suspens, coups de théâtre, et autres effets de mise en relief
}

\author{
Le Draoulec, Anne \\ CLLE-ERSS(UMR5263), CNRS \& Université de Toulouse-Le Mirail \\ draoulec@univ-tlse2.fr
}

\section{Introduction}

La subordination dite « inverse » (en « souvenir », selon Wilmet, 2010, du cum inversum de la grammaire latine) est un phénomène bien connu en linguistique, et déjà largement exploré. Le seul quand (ou lorsque) inverse $^{1}$ (dont (1) est l'exemple prototypique) a fait l'objet de nombreuses études, parmi lesquelles on mentionnera Olsson, 1971; Chétrit, 1976; Borillo, 1988 ; Declerck, 1997² ; Vogeleer, 1998 ; Benzitoun, 2006 :

(1) Il dormait profondément, quand (soudain) un cri le réveilla

Parfois aussi (cf. Maurel, 1992; Combettes, 2011), quand inverse est examiné parmi d'autres constructions plus ou moins proches, telles les constructions en à peine (ou pas plus tôt/sitôt)... que (cf. (2)), les relatives continuatives (cf. (3)), certains types de subordonnées consécutives (cf. (4)) ou hypothétiques (cf. (5)) $)^{3}$ :

(2) À peine Priscilla était-elle sortie qu'il se mit à pleuvoir (cf. Maurel, 1992: 72)

(3) Il ouvrit la porte, qu'il referma aussitôt (ibid. : 78)

(4) Il écrivit beaucoup, si bien qu'il passa pour un écrivain (ibid. : 76)

(5) il eût longtemps juré inutilement si l'officieux Colomne ne fût arrivé à son secours (Histoire de Nogaret et de Mariane (1670) (cf. Combettes, $2011: 90$ )

Les divers types de construction inverse ont pour point commun de présenter une distribution particulière des informations en termes de principal vs secondaire : à la principale - syntaxique - l'expression de l'idée secondaire (ou accessoire, circonstancielle), à la subordonnée l'expression de l'idée principale. La notion de subordination inverse apparaît ainsi comme un mixte de considérations syntaxiques et sémantiques. Ou comme le dit très bien Combettes $(2011: 84)$ :

[...] la dénomination, sans doute peu satisfaisante, de « subordination inverse », si elle renvoie au domaine syntaxique par l'emploi du terme de "subordination », réfère davantage au versant sémantique par celui d' " inverse ». Il s'agit en effet, par le choix de ce terme, de mettre en avant la discordance qui s'établit, dans cette construction, entre les marques formelles de la subordination et les relations de sens.

L'insatisfaction suscitée par une appellation mêlant syntaxe et sémantique, telle qu'elle est dite par Combettes, est de fait sans cesse rappelée - on y reviendra (section 2). Pour l'instant, on insistera surtout sur les effets de sens dont s'acompagne la discordance ainsi pointée : effets diversement qualifiés (à propos, plus particulièrement, du quand inverse) d'effets de « surprise », « interruption », « suspens » ou « suspension », et qui sont selon Vogeleer (1998) proprement constitutifs de l'interprétation inverse, dans la mesure où «c'est cet effet [de surprise] qui distingue le quand inverse du quand canonique (p. 80) ». Sechehaye (1926) parlait déjà, en ce sens, de «quand de péripétie ». Combettes (2011) mentionne plus généralement pour sa part, à propos des deux types de construction illustrés par (1) et (2), un effet de «mise en relief» allant de pair avec l'opposition aspectuelle bien connue entre aspect global dans la subordonnée (prototypiquement au passé simple) vs sécant dans la principale (prototypiquement à l'imparfait). Une telle opposition aspectuelle en effet (communément décrite, également, en termes de perfectif versus imperfectif) contribue à ce que, au niveau textuel, le «procès de la subordonnée [... ] se détache $[\ldots]$ sur le fond constitué par le procès non borné qui ouvre l'énoncé » (p. 84). À la discordance 
évoquée plus haut s'associe ainsi, sur le plan discursif, un écart par rapport à la répartition habituelle des plans - cf. là encore Combettes, qui voit dans la subordination inverse
une disposition textuelle particulière, qui s'écarte des tendances générales de la répartition des plans, tendances qui font coïncider premier plan et proposition régissante, alors que les subordonnées sont plutôt réservées à l'expression du second plan. (pp. 84-85)

Notre objectif, dans la présente étude, n'est pas d'ajouter une nouvelle approche théorique de la notion de subordination inverse, ou plus spécifiquement du quand inverse, mais de mettre en évidence qu'une construction jusqu'ici peu explorée devrait, selon nous, être rangée sous la même catégorie de l' « inversion » (ou quelque autre nom qu'on voudra lui donner). Il s'agit de la construction en avant que ou avant de que nous avons mise au jour et désignée comme «construction assertive » (cf. Le Draoulec, 2005, 2006) : une construction où avant que ou avant de (désormais $A V$ lorsque nous souhaiterons désigner indistinctement les deux formes, i.e. avec ou sans réduction infinitive de la subordonnée), au lieu de jouer son rôle classique de "déclencheur de présupposition", permet une relation de succession temporelle entre deux propositions également assertées, comme en (6) :

(6) Âgée de 47 ans, la victime a été grièvement blessée jeudi, avant de décéder des suites de ses blessures. (liberation.fr, 6 avril 2012)

Dans ce type d'exemple, les événements reliés par $A V$ apparaissent sur le même plan, i.e. sans que le second soit perçu comme participant à la localisation temporelle du premier. Le rôle de $A V$ en construction assertive (par raccourci, $A V$ assertif) le rapproche ainsi de celui d'un connecteur temporel tel que puis.

En explorant cette construction assertive, nous l'avions mise en regard, déjà, avec la construction en quand inverse, dont elle partage et le caractère assertif et la propriété d'avancement narratifi ${ }^{4}$. Nous insistions cependant sur la différence entre les deux types de construction, en ce sens que $A V$ assertif ne déclencherait pas d'inversion des premier et second plans (les deux propositions qu'il relie apparaissant plutôt sur un même plan), ni ne s'accompagnerait des effets de sens remarquables (suspens, surprise, interruption) par lesquels quand inverse se distingue : raison pour laquelle, probablement, l'existence de $A V$ assertif était restée méconnue, alors que quand inverse concentrait toutes les attentions.

Or on rencontre des exemples où un $A V$ assertif déclenche des effets normalement associés à quand inverse : surprise (littéralement illustrée en (7)) éventuellement couplée à un effet de suspens, avec interruption soudaine (cf. en (7) encore l'usage de soudain) d'une situation ${ }^{5}$ en cours :

(7) Mais c'est surtout la continuation éclatante de l'œuvre polanskienne, le prolongement d'une vision du monde où le pire paraît peu à peu s'éloigner, comme un mauvais rêve, avant de revenir, soudain, envahir la réalité. La surprise finale et ses retentissements rappellent cette étrange formule de Lacan : "La paranö̈a, c'est la vérité. »(Télérama, 3 mars 2010)

La presse, en particulier, use très régulièrement de ce procédé suspensif pour introduire un moment clé (basculement de l'intrigue, ou dénouement) dans des résumés de films (cf. (8)), de livres (cf. (9)), ou dans des récits de faits divers (cf. (10)) :

(8) Repéré par un chasseur de têtes, un jeune ingénieur se voit promis à une belle carrière dans une société prestigieuse. Avant de comprendre qu'il a vendu son âme au diable... (Télérama, 14 mars 2012)

(9) [...] dans Un lieu incertain comme dans bien d'autres, [...] frères et/ou fils ennemis n'ont de cesse de s'affronter sur fond de traîtrise, et toujours les nerfs se tendent, remontés comme des horloges, avant de se relâcher, suivant le bon vieux processus de la catharsis. (Le Magazine Littéraire, juillet-août 2008)

(10) Un commerçant marseillais, patron d'un débit de tabac, a été retenu en otage pendant six jours avant d'être libéré mardi matin par la police dans une ferme près d'Aix-en-Provence, a-t-on appris de source proche de l'enquête. (Libération.fr, 14 décembre 2010)

Nous nous proposons ici de porter plus loin l'analyse de $A V$ assertif en nous appuyant sur de nombreux exemples attestés du même type qui, au fur et à mesure que nous les avons accumulés (au fil de diverses lectures personnelles), nous ont convaincue qu'il ne s'agit pas là d'un phénomène anecdotique, mais bien 
plutôt d'effets de sens massifs et réguliers, quoique non systématiques. En focalisant notre attention sur ces effets de sens et les configurations auxquelles ils sont associés, nous serons amenée à réévaluer notre comparaison entre quand inverse et $A V$ assertif, et la façon dont celui-ci, au final, vient selon nous élargir la gamme des constructions de subordination inverse.

\section{Préliminaires : $A V$ assertif dans le sillage de quand inverse}

Commençons par revenir sur la difficulté que pose l'appellation de «subordination inverse » qui mêle, ainsi que nous l'avons dit, des considérations syntaxiques et sémantiques. Ce mélange en fait selon certains (en particulier des syntacticiens, cf. Delofeu, 1988 ; Benzitoun, 2006 ; Corminboeuf, 2007) une aberration terminologique, dissimulant mal une circularité du raisonnement: continuer de regarder comme marque de subordination un que ou un quand dont tout montre que la proposition qu'il introduit n'est pas syntaxiquement régie est ainsi, selon Benzitoun (2006:97), aussi absurde que de «parler de "subordonnée non subordonnée" ». Et pourtant, malgré tous ses défauts, la dénomination de « subordination inverse » survit et continue d'être communément utilisée, comme c'est souvent le cas des notions les plus contestées en linguistique. Nous nous accommoderons également pour notre part de cette appellation, à la manière de Combettes (2011) - c'est-à-dire en en reconnaissant les limites, et en nous focalisant sur les aspects sémantiques et discursifs liés à l'opposition des premier et second plans. Montrer que le $A V$ assertif se rapproche du quand inverse, ce sera donc essentiellement, dans notre perspective, montrer qu'il partage des caractéristiques similaires à celles de quand inverse en termes de répartition des plans. Nous laisserons ainsi de côté les aspects proprement syntaxiques de ces deux constructions, nous contentant de renvoyer à Benzitoun (2013), qui en propose une étude comparée éclairante : étude où est mise en évidence leur autonomie syntaxique respective (en termes d'absence de rection). En ce qui concerne les aspects sémantiques et énonciatifs, on ne reviendra pas sur l'autonomie énonciative - suffisamment démontrée ailleurs - des propositions introduites par un quand inverse. On rappellera brièvement en revanche, à la suite de Le Draoulec (2005, 2006), les caractéristiques essentielles de la construction avec un $A V$ assertif, en comparaison avec la construction (classiquement) présuppositionnelle. Pour ce faire on opposera à l'exemple (6) de l'introduction (répété ci-dessous) les exemples (11) et (12) :

(6) Âgée de 47 ans, la victime a été grièvement blessée jeudi, avant de décéder des suites de ses blessures.

(11) Certaines filles n'avaient jamais chaussé de patins avant de démarrer le derby. (Rue 89, 21octobre 2012)

(12) Christian Prudhomme, directeur du Tour de France, a eu un discours fort sur le dopage devant le Palais des Congrès, avant de présenter le parcours. C'est encourageant mais pour l'instant insuffisant. (Rue 89, 24 octobre 2012)

En (11) ou (12), on présuppose réalisés les événements dénotés par les filles ont démarré le derby ou le directeur du Tour de France a présenté le parcours, et c'est par rapport au temps de chacun de ces événements qu'est déterminé le temps de référence de la proposition principale. Le propre temps de référence de la subordonnée temporelle n'est selon Hamann (1989) que « secondaire », au sens où il ne fait que participer au calcul du temps de référence "courant», celui de la principale (sans jamais apparaître lui-même comme temps de référence courant). En d'autres termes, la subordonnée ne participe pas à l'avancement de la narration. Et, si elle apparaît, dans chacun de nos deux exemples, en position finale, elle pourrait aussi bien être déplacée à l'initiale.

En (6) en revanche, la proposition introduite par avant de a une autonomie énonciative qui correspond à celle d'une assertion. Elle fait clairement progresser le récit des événements, en mettant à jour le temps de référence courant. On notera que le déplacement en position initiale s'avère, en ce cas, impossible (à moins d'un changement notable d'interprétation) :

(6') ??Avant de décéder des suites de ses blessures, la victime, âgée de 47 ans, a été grièvement blessée jeudi.

La postposition de la subordonnée introduite par $A V$ est, de fait, la seule condition nécessaire - quoique non suffisante - à la construction assertive (comme c'est également le cas avec un quand inverse). Deux autres conditions très nettement favorables sont, d'une part, le détachement de la subordonnée par une 
marque de ponctuation, allant de la simple virgule (comme, le plus souvent, devant un quand inverse) à une ponctuation plus forte (tiret, point, points de suspension); et d'autre part, la conservation du sujet entre la principale et la subordonnée, avec, le plus souvent, une réduction infinitive de la seconde (ce qui n'est évidemment pas le cas avec un quand inverse) ${ }^{7}$. Ces dernières conditions ne sont cependant ni suffisantes, ni nécessaires, ainsi que le montrent les exemples suivants où la construction assertive s'accommode, en (13), de l'absence de ponctuation devant avant de, ou en (14), du changement de sujet entre principale et subordonnée ${ }^{8}$ :

(13) Quelques mois plus tard, il [le fils d'Andréas Papandréou] fut rattrapé par l'explosion de la dette avant de sombrer dans des abîmes d'impopularité. (Libération, 4 novembre 1011)

(14) Le récit plonge parfois dans de sérieux temps morts. Avant que les comédiens, tous formidables, ne le fassent rebondir vers des sommets d'émotion. (Télérama, 10 février 2010)

Le seul critère permettant d'attester qu'on est en présence d'une construction assertive est le critère d'enchaînement sur le contenu de la subordonnée - c'est-à-dire la non-application de la «loi d'enchaînement » ainsi définie par Ducrot pour la présupposition :

Lorsque A est enchaîné, par une conjonction de coordination ou de subordination, ou par un lien logique implicite, à un autre énoncé $\mathrm{B}$, le lien ainsi établi entre $\mathrm{A}$ et $\mathrm{B}$ ne concerne jamais ce qui est présupposé, mais seulement ce qui est posé par A et par B. (Ducrot 1991: 81)

Alors qu'il est impossible, donc, d'enchaîner sur le contenu présupposé d'une subordonnée temporelle " ordinaire ", au sens de classiquement présuppositionnelle, on peut enchaîner sans difficulté sur une proposition introduite par un $A V$ assertif (aussi bien que par un quand inverse) ${ }^{9}$. On pourrait ainsi poursuivre (6) de la façon suivante :

(6") Âgée de 47 ans, la victime a été grièvement blessée jeudi, avant de décéder des suites de ses blessures. Pourtant, tout avait été tenté pour la sauver.

où le lien de concession établi par Pourtant (Pourtant, tout avait été tenté pour la sauver) porte sur le contenu de la proposition introduite par avant de.

On trouve d'ailleurs des exemples où la suite du discours, telle qu'elle est effectivement donnée, illustre précisément cette possibilité d'enchaînement. C'est le cas en (15) ci-dessous, où Puis il a insisté sur le fait que Strauss-Kahn avait démissionné de son poste enchaîne sur Avant de demander pourquoi :

(15) Le juge a également relevé le fait que DSK et ses avocats n'avaient pas choisi de mettre en avant cet argument de l'immunité lors de la procédure pénale. Avant de demander pourquoi. Puis il a insisté sur le fait que StraussKahn avait démissionné de son poste avant d'être placé face à cette plainte civile. (Libération, 28 mars 2012) ${ }^{10}$

La façon dont la construction assertive façonne le contexte environnant en autorisant l'enchaînement ne constitue pas cependant une condition, mais plutôt un effet de la construction - un effet dont l'authentification reste, qui plus est, aléatoire, l'enchaînement pouvant être ou non effectivement attesté ${ }^{11}$.

\section{Effets de sens}

Nous en arrivons enfin à la question qui va nous occuper plus particulièrement des effets de sens : effets systématiques voire définitoires pour le quand inverse, a priori moins systématiques pour le $A V$ assertif. Comme annoncé en introduction, c'est sur ce point que nous semblaient diverger les fonctionnements des deux constructions; et c'est sur ce point que nous nous appliquerons à montrer que la convergence est plus grande qu'il ne nous avait d'abord semblé. Car, si l'opposition aspectuelle caractéristique du quand inverse n'existe pas dans le cas du $A V$ assertif, il apparaît que d'autres configurations se mettent en place très régulièrement, susceptibles d'amener à des résultats comparables. On montrera qu'il s'agit en particulier de configurations où les propositions principale et subordonnée présentent une relation de contraste (section 3.1.), ou encore où la situation décrite dans la subordonnée vient clore une énumération (section 3.2.). 
Précisons que nous désignerons, dans les sections qui suivent, par $\mathrm{P}_{1}$ et $\mathrm{P}_{2}$ (éventuellement $\mathrm{P}_{\mathrm{n}}$ et $\mathrm{Pn}_{\mathrm{n}}$ ) les propositions reliées par $A V$ - ce qui aura le mérite de gommer les appellations syntaxiquement contestables de propositions principale et subordonnée - et par $\mathrm{S}_{1}$ et $\mathrm{S}_{2}\left(\mathrm{~S}_{\mathrm{n}}\right.$ et $\left.{ }_{\mathrm{Sn}+1}\right)$ les situations qu'elles décrivent.

\subsection{Contraste et effet de renversement (ou d'intensification) de situation}

Le premier type de configuration auquel se conforme un grand nombre de nos exemples comporte une relation d'opposition sémantique entre $S_{1}$ et $S_{2}$ (fondant une relation discursive de contraste entre $P_{1}$ et $\left.\mathrm{P}_{2}{ }^{12}\right)$. Nos exemples $(7)$ à $(10)$, ou encore (14), cités plus haut, en étaient déjà des illustrations. On l'observera plus précisément à propos de l'exemple (14) ainsi que des exemples (16) et (17), très proches de (14) à la fois par le schéma argumentatif (critique d'un film « en deux temps »), et par l'usage qui est fait de $A V$ (introduisant le « second temps ») :

(14) Le récit plonge parfois dans de sérieux temps morts. Avant que les comédiens, tous formidables, ne le fassent rebondir vers des sommets d'émotion. (Télérama, 10 février 2010)

(16) Cette conquête simiesque de l'espace fait sourire dans le premier quart d'heure avant de virer à la mauvaise science-fiction. (Télérama, 22 octobre 2008)

(17) Daybreakers, des Australiens Michael et Peter Spierig, commence plutôt bien, avec un mélange inventif de fantastique et d'anticipation (le monde moderne a été «adapté » à la condition nocturne de l'espèce dominante), avant de s'autodétruire plus vite que Dracula un jour de canicule. Scènes d'action mal fichues, intrigue bâclée... Dommage. (Télérama, 3 mars 2010)

Dans ces exemples, le remplacement de $A V$ par un connecteur temporel tel que puis, dont on soulignait en introduction qu'il a un rôle proche de celui du $A V$ assertif, ne serait pas satisfaisant, en ce qu'il occulterait la prééminence donnée à chaque fois à $\mathrm{P}_{2}$. Dans chacun de ces exemples, en effet, $\mathrm{P}_{1}$ argumente en faveur d'un jugement soit négatif (cf. (14)), soit positif (cf. (16)-(17)) sur le film, et $\mathrm{P}_{2}$ en faveur du jugement inverse, soit positif, soit négatif; et, dans tous les cas, c'est le dernier argument qui l'emporte. D'où un rapprochement possible ici de $A V$ avec mais, dont la force argumentative a été très précisément décrite par Ducrot, dans une analyse dont on rappellera simplement le principe essentiel :

Lorsqu'on coordonne par mais deux propositions $p$ et $q$, on ajoute à $p$ et à $q$ les deux idées suivantes. D'abord, qu'une certaine conclusion $\mathrm{r}$, que l'on a précisément dans l'esprit, et que le destinataire peut retrouver, serait suggérée par $p$ et infirmée par $q$ : autrement dit, $p$ et $q$ ont, par rapport à r, des orientations argumentatives opposées. Ensuite, que $q$ a plus de force contre $\mathrm{r}$ que $p$ n'en a en sa faveur : de sorte que l'ensemble $p$ mais $q$ va dans le sens de non-r. (Ducrot, $1980: 11-12$ ).

De la même façon que mais, $A V$ en (14), (16) ou (17) introduit une proposition $\mathrm{P}_{2}$ dotée d'une force argumentative supérieure à celle de $\mathrm{P}_{1}$ : on conclura de (14) que le film est «plutôt bon », et de (16) ou (17) qu'il est «plutôt mauvais ». (16), par exemple, pourrait être paraphrasé par (16') (où le rôle d'avancement temporel de $A V$, dont mais est dépourvu, est pris en charge par l'ajout d'un adverbe tel qu'ensuite ou après) :

(16') Cette conquête simiesque de l'espace fait sourire dans le premier quart d'heure mais ensuite/après elle vire à la mauvaise science-fiction.

Cette force argumentative supérieure donnée au dernier terme permet l'expression d'un « retournement de situation », ou «volte-face », « coup de théâtre », qu'on trouve littéralement illustrés en (18), (19) ou (20) :

(18) [Elle est] soudain bombardée reine, et [...] essuie bien des humiliations de la part de son époux infidèle et de sa belle-mère sévère, avant de retourner la situation et l'opinion à son avantage. (Télérama, 23 novembre 2005)

(19) Ira ? Ira pas ? Rupert Murdoch a passé la journée d'hier à jouer avec les nerfs des députés britanniques. «Invité» à venir témoigner mardi devant un comité de la Chambre des Communes pour s'expliquer sur l'affaire des écoutes illégales de News of the World, le magnat australo-américain de 80 ans a d'abord catégoriquement décliné l'invitation. Avant de se raviser. Sauf volte-face de dernière minute ${ }^{13}$, il devrait donc se présenter au Parlement accompagné de son fils James et de Rebekah Brooks [...]. (Libération, 15 juillet 2011) 
(20) Critiqué par ses alliés à la suite de sa prestation ratée lors de son duel télévisé avec Romano Prodi, en retard dans les sondages à trois semaines du scrutin, Berlusconi a en tout cas réussi à reprendre la vedette. Alors que, vendredi, le leader de la gauche, Romano Prodi, avait été accueilli avec intérêt mais sans enthousiasme particulier par le patronat italien, Berlusconi, attendu samedi à Vicence, avait d'abord déclaré forfait «en raison de problèmes de dos». Avant de se raviser : "Quand j'ai entendu ce que vous a dit Prodi, j'ai dit aux médecins de me faire une infiltration, il fallait absolument que je vienne», a-t-il justifié, très content de son coup de théâtre. (Libération, 20 mars 2006)

On notera, en (19), la question initiale Ira? Ira pas? qui résume très bien l'alternative dont la construction en $A V$ se fait l'écho; et en (19) toujours et en (20), l'utilisation du verbe se raviser, pour l'expression d'un retournement/renversement de situation correspondant à un retour en arrière - retour en arrière qu'on trouve explicité par l'usage de l'expression machine arrière en (21) :

(21) Auparavant, Ryanair avait aussi émis l'idée de faire payer davantage les passagers les plus corpulents. Avant de faire machine arrière devant le tollé déclenché par cette proposition. (Libération, 7 juillet 2009)

On mentionnera encore, pour continuer d'illustrer et préciser cette idée de renversement de situation, les exemples (22), (23) et (24) :

(22) Le 26 janvier, le candidat a pourtant annoncé à la télévision qu'il la maintiendrait [la loi sur le «service garanti »], précisant : "Il y a une loi, elle marche, je ne perds pas mon temps. »

L'avant-veille, à l'Assemblée, les quelques députés socialistes présents avaient "perdu leur temps », eux, en votant contre l'extension du «service garanti» au transport aérien. Avant que le candidat socialiste ne l'approuve en 2017 ? (Canard enchaîné, 11 avril 2012)

(23) $[\ldots]$ ceux qui le connaissent sont persuadés qu'il a dû paniquer qu'une bombe sexuelle comme Emma tombe amoureuse de lui. Avant d'être flatté. (Paris-Match, 20 janvier 2011)

(24) On n'a qu'à regarder pour voir que l'être humain est divisé en deux parties symétriques dans le sens de la longueur. Et, à l'intérieur, de même notre fonction est desservie par des organes gémeaux [...]. Mais avec le cou, [...] toute cette architecture aux étages successifs aboutit à l'unité. Avant que la cervelle avec son double lobe, le visage avec ses deux yeux, ses deux oreilles, [...] ne donne à notre dualité le sceau suprême de la composition (Paul Claudel, Commentaires et exégèses)

En (22), le renversement de situation, de perdre son temps en votant contre à approuver, est immédiatement lisible. L'exemple a de plus le grand mérite de souligner, s'il le fallait encore, l'autonomie énonciative de la proposition $\mathrm{P}_{2}$, du fait du mode interrogatif auquel elle apparaît. Par rapport à ce cas de figure bien tranché, l'exemple (23) apporte une nuance. Il met, en effet, en évidence que le « renversement » n'exige pas une opposition directe (une " inversion ») des situations (puisque paniquer ne s'oppose pas directement à être flatté), mais peut plus généralement s'appuyer sur un infléchissement de la situation, de telle sorte que $\mathrm{S}_{1}$ a des conséquences opposées à celles de $\mathrm{S}_{2}$ : ce qui, là encore, nous ramène au parallèle entre $A V$ et mais (cf. il a dû paniquer mais ensuite/après il a été flatté).

Quant à l'exemple (24), il a ceci de remarquable que $\mathrm{P}_{2}$ introduit par avant que est précédé d'un $\mathrm{P}_{1}$ luimême introduit par mais : alors que mais $\mathrm{P}_{1}$ argumente en faveur de l'unité chez l'être humain, avant que $\mathrm{P}_{2}$ permet de dépasser cette conclusion (provisoire), pour revenir à l'idée de dualité telle qu'elle avait été préalablement affirmée. C'est-à-dire plus généralement que, dans le mouvement argumentatif, non seulement $A V$ joue un rôle similaire à celui de mais mais encore l'un et l'autre peuvent se succéder avec le même pouvoir de contradiction - celui qui l'emporte étant celui qui apparaît en dernier.

La comparaison de $A V$ avec mais (plus précisément mais ensuite ou mais après), cependant, ne rend pas compte des effets de suspens et de surprise provoquée par la résolution du suspens, tels qu'ils s'associent au renversement de situation introduit par $A V$ : association glosée plus haut par les expressions volte-face ou coup de théâtre, et qu'on pourrait également exprimer à l'aide d'une paraphrase où serait introduit un adverbe tel que finalement (cf. mais après, finalement, il a été flatté / et puis finalement, il a été flatté). Suspens et surprise sont liés à l'usage de $A V$ assertif aussi bien qu'à celui de quand inverse - à une différence de systématicité près puisqu'avec $A V$ assertif, des conditions particulières comme celles de contexte oppositif sont requises. Avec $A V$ assertif, le suspens peut être renforcé par une insistance sur la durée, la prolongation de $\mathrm{S}_{1}$ venant faire pendant à opposition aspectuelle régulière entre $\mathrm{S}_{1}$ et $\mathrm{S}_{2}$ lorsqu'il s'agit de quand inverse. C'était le cas dans plusieurs des exemples mentionnés plus haut (cf. les diverses 
mentions de paraît peu à peu s'éloigner en (7), pendant six jours en (10), ou essuie bien des humiliations en (18)). C'est également le cas dans les exemples ci-dessous, où la durée de $\mathrm{S}_{1}$ est soulignée par le syntagme adverbial plusieurs années durant (en (25)), par le syntagme verbal passer des heures à (en (26)), ou par l'utilisation de points de « suspension » (justement) (en (27)) :

(25) [...] Globalement, j'ai mis du temps à trouver ce rythme et à l'accepter. Plusieurs années durant, je me suis satisfaite d'une certaine inertie. Avant de comprendre qu'on doit se donner un coup de pied au cul pour saisir sa chance. (Libération, 29 avril 2006)

(26) Leur travail sériel, têtu, obsessionnel, donne le vertige. On rit souvent devant l'uniformité des extravagances, on passe des heures à scruter ces similitudes parfois grotesques. Avant de se retrouver à son tour épinglé. Là, on rigole moins. (Télérama, 8 octobre 2008)

(27) Elle reçoit finalement une réponse de la préfecture la veille de Noël. Sa demande est acceptée. Soulagée, elle se met à la recherche d'un nouvel emploi, cale des entretiens d'embauche... Avant d'être à nouveau coupée dans son élan. (Libération, 14 mars 2012)

Mais en dehors même de ces cas où le suspens est soutenu par des marqueurs particuliers, il s'associe déjà au simple temps de séparation - induit par $A V$ comme marqueur d'antériorité - entre la réalisation de $\mathrm{S}_{1}$ et celle de $\mathrm{S}_{2}$.

Dans la continuité de nos exemples de renversement de situation, nous ferons figurer ici encore un autre type d'exemples qui, s'ils ne présentent pas à proprement parler une opposition sémantique entre les situations $S_{1}$ et $S_{2}$, présentent entre elles une gradation d'intensité ; de telle sorte que l'effet de sens, s'il n'est pas de renversement de situation, correspond encore à une prééminence donnée à $S_{2}$ sous forme de ce que nous désignons, faute de trouver un meilleur terme, comme "intensification ». Nos exemples (6) ou (13) en étaient déjà des illustrations. Les exemples ci-dessous vont également en ce sens, avec une gradation claire entre fédérer une petite communauté de lecteurs et déclencher une véritable fièvre autobiographique (en (28)), ou entre se cacher derrière son foulard et disparaître (en (29)) :

(28) Lancé en 2006, le «smithmag» a réussi à fédérer une petite communauté de lecteurs avec ses ateliers d'écriture. Avant de déclencher une véritable fièvre autobiographique grâce à cette idée toute simple : «Ecrivez vos mémoires en six mots». En quelques jours, plus de onze mille contributions affluent. (Télérama, 28 mai 2008)

(29) Mal à l'aise, la femme qui semblait il y a quelques instants si sûre d'elle se cache derrière son foulard, avant de disparaitre. Une tradition locale, les femmes de l'Haryana ayant l'habitude de dissimuler leur visage devant des hommes âgés ou extérieurs au cercle familial. (Libération, 12 mars 2010)

On remarquera pour ces exemples qu'ils pourraient encore être analysés comme présentant une relation discursive de contraste entre $\mathrm{P}_{1}$ et $\mathrm{P}_{2}$ (surtout si, en (28), on tient également compte de la suite donnée à $\mathrm{P}_{2}$ : En quelques jours, plus de onze mille contributions affluent). $\mathrm{P}_{1}$ et $\mathrm{P}_{2}$ en effet ont des implications ou implicatures contradictoires, du type : «peu de gens sont concernés» / «beaucoup de gens sont concernés » (pour (28)), ou « le visage de la femme est encore partiellement visible » / « son visage n'est plus du tout visible (puisqu'elle est partie) » (pour (29)). Nous ne sommes pas sûre que cette analyse soit tout à fait pertinente ${ }^{14}$ ni qu'elle pourrait s'appliquer à tous les cas de gradation d'intensité. Nous conserverons néanmoins, par approximation, ce terme de «contraste » pour désigner des configurations où $\mathrm{P}_{1}$ et $\mathrm{P}_{2}$ décrivent des situations en relation d'opposition aussi bien que de gradation d'intensité.

L'exemple (30) enfin est particulièrement intéressant dans la mesure où il semble allier effets d'intensification et de renversement de situation - faire son petit effet apparaissant aussi bien dans le prolongement du salut du travail de la maire (autrement dit comme un salut « optimal»), qu'en rupture avec ce même salut (par ouverture à des perspectives nationales plutôt que municipales) :

(30) Le premier habitant à prendre le micro est un fan absolu. Il salue le travail de la maire pendant trois minutes avant de faire son petit effet: "Ce que vous faites si bien à Lille, ce que vous faites si bien à la communauté urbaine, je voudrais bien vous le voir faire ailleurs». (Libération, 23 juin2011)

Entre les deux types d'effets, il ne nous semble ainsi pas toujours nécessaire de trancher. 


\subsection{Fin d'énumération et effet de clôture}

Revenons un instant sur les exemples (26) et (27) de la section qui précède. Ils ont ceci de particulier que le retournement de situation introduit par $A V$ ne coïncide pas proprement avec une opposition entre $\mathrm{S}_{1}$ et $\mathrm{S}_{2}$, i.e. entre passer des heures à scruter ces similitudes [chez les autres] et se retrouver à son tour épinglé pour (26), ou caler des entretiens d'embauche et être à nouveau coupée dans son élan pour (27). L'opposition, en (26), se joue plutôt entre les blocs rire souvent / passer des heures à scruter ces similitudes d'une part, et se retrouver à son tour épinglé / rigoler moins d'autre part; et en (27), entre le bloc se mettre à la recherche d'un nouvel emploi / caler des entretiens d'embauche d'une part, et être à nouveau coupée dans son élan d'autre part. C'est-à-dire que, s'il relie formellement $\mathrm{P}_{1}$ et $\mathrm{P}_{2}, A V$ participe à la mise en place d'une relation de contraste qui dépasse les seules situations $\mathrm{S}_{1}$ et $\mathrm{S}_{2}$ telles qu'elles sont strictement décrites par $\mathrm{P}_{1}$ et $\mathrm{P}_{2}$.

Nous allons, dans ce qui suit, nous intéresser à des configurations de ce type, et plus particulièrement du type de (27), où la proposition introduite par $A V$ arrive à la suite de deux, trois, voire plus - toute une série de - propositions préalables, auxquelles elle apporte une forme de conclusion, de clôture. Une clôture qui, en (27), reste associée à une relation de contraste avec l'ensemble qui précède ; mais on verra que ce n'est pas nécessairement le cas.

Examinons ainsi les exemples (31) à (38), où la proposition introduite par $A V$ est la dernière d'une série de propositions :

(31) En remontant le courant, on s'aperçoit d'ailleurs que cette disparition [du mot « rupture » dans le discours de Sarkozy] ne s'est pas faite en un jour: "Dans un premier temps, [Nicolas Sarkozy se rend compte que le mot fait peur à une partie de son électorat, $]_{\mathrm{P} 1}$ analyse Calvet, alors [il lui colle l'adjectif « tranquille », emprunté à Mitterand. $]_{\mathrm{P} 2}$ Puis [il affirme que l'important n'est pas de savoir " avec qui », mais « avec quoi » il rompt... $]_{\mathrm{P} 3}$ avant d'[abandonner complètement l'idée, sans que personne ne lui demande d'explications $]_{\mathrm{P} 4}$ ». Sauf Ségolène Royal, [...] (Télérama, 14 février 2007)

(32) [Il ronge le grillon de l'intérieur, $]_{\mathrm{P} 1}$ puis [prend les commandes de son cerveau $]_{\mathrm{P} 2}$ avant de [le pousser au suicide : $]_{\mathrm{P} 3}$ ce redoutable ver manipulateur en dit long sur les modes d'action des parasites. (Libération, 29 avril 2006)

(33) Durant douze jours, les huit candidats au suicide sentimental vivront au milieu de vingt-deux célibataires, dans la plus parfaite «harmonie »: [ils résisteront, $]_{\mathrm{P} 1}$ puis $[\text { craqueront, }]_{\mathrm{P} 2}$ [regretteront, $]_{\mathrm{P} 3}$ avant de [tirer un trait.] $]_{\mathrm{P} 4}$ (Télérama, 7 juillet 2004)

(34) [On y voit le docteur Shutt, d'humeur particulièrement ronchonne, s'éreinter au golf, $]_{\mathrm{P} 1}$ puis [s'engueuler avec son patron, $]_{\mathrm{P} 2}$ puis [rudoyer un piano mécanique $]_{\mathrm{P} 3}$ avant de [s'effondrer, victime d'une rupture d'anévrisme. $]_{\mathrm{P} 4}$ (Télérama, 3 septembre 2003)

(35) [L'oncle et la nièce, farouchement enlacés devant la porte ouverte du compartiment du train lancé à vive allure, se retiennent, $]_{\mathrm{P} 1}\left[\mathrm{~s}^{\prime} \text { agrippent, }\right]_{\mathrm{P} 2}$ [se poussent, $]_{\mathrm{P} 3}$ avant de [tomber dans le vide. $]_{\mathrm{P} 4}$ Pour Charlie, le miracle a eu lieu. (Télérama, 22 février 2012)

(36) Il y a des gens [qui attendent, $]_{\mathrm{P} 1}$ [qui temporisent, $]_{\mathrm{P} 2}$ [qui se font une opinion, $]_{\mathrm{P} 3}$ avant, finalement, de [ne rien faire.] $]_{\mathrm{P} 4}$ Pas Lucie [Aubrac]. Elle a toujours milité contre. (Charlie Hebdo, 28 décembre 2005)

(37) Mais [dans cette affaire, l'Elysée a tergiversé pendant des mois, $]_{\mathrm{P} 1}$ [tiré à vue sur la patronne, $]_{\mathrm{P} 2}$ [entretenu les rivalités les plus déraisonnables $]_{\mathrm{P} 3}$ avant de [trancher sans la moindre explication ni nouvelle feuille de route. $]_{\mathrm{P} 4}$ (Libération, 18 juin 2011)

(38) [Il y a celles qui s'attachent au metteur en scène et à son projet, $]_{\mathrm{P} 1}$ [celles qui prétendent « s'éclater » devant sa caméra, $]_{\mathrm{P} 2}[\text { celles qui le voient en père, }]_{\mathrm{P} 3}[\text { en confident, }]_{\mathrm{P} 4}[\mathrm{en} \text { psy, }]_{\mathrm{P} 5}$ [en sexologue.... $]_{\mathrm{P} 6}$ Avant, pour certaines, de [le haïr. $]_{\mathrm{P} 7}$ (Télérama, 13 septembre 2006)

Ces exemples suivent des schémas qui se recoupent. Nous choisissons cependant d'en faire figurer un certain nombre, au risque de la répétition, afin de mettre en évidence la régularité du phénomène auquel nous nous intéressons. Précisons que, dans nos exemples, les propositions que nous incluons dans une même série (et que nous segmentons comme $\mathrm{P}_{1} \ldots, \mathrm{P}_{\mathrm{n}}, \mathrm{P}_{\mathrm{n}+1}$ ) ne sont pas nécessairement des propositions complètes (c'est, en fait, uniquement le cas en (31)), mais peuvent être réduites à un syntagme verbal, voire à un syntagme nominal ou prépositionnel (cf. (38)). Sur le plan syntaxique et discursif, on peut arguer qu'on a affaire à des syntagmes qui, par ellipse, renvoient à des propositions plus complètes. Nos 
choix de segmentation, pour autant, restent très certainement contestables ${ }^{15}$. Mais nous nous y tiendrons dans la mesure où ils permettent de mettre en évidence le phénomène d'énumération qui, de façon incontestable, est ici dans tous les cas à l'œuvre.

Pour chacun de ces exemples nous semble-t-il, il serait possible, plus ou moins artificiellement, de trouver un motif d'opposition entre $S_{n+1}$ et l'ensemble des situations $S_{1}-S_{n}$ qui précèdent. Dans l'exemple (38) en particulier, une opposition s'établit assez facilement entre hä̈r le metteur en scène et toute la palette d'émotions/relations plus positives qui précèdent. Dans les autres exemples, le motif d'opposition est plus compliqué à récupérer - même si l'on peut toujours avancer que abandonner complètement l'idée (en (31)) / pousser au suicide (en (32)) / tirer un trait (en (33)) / s'effondrer (en (34)) / tomber dans le vide (en (35)) / ne rien faire (en (36)) / trancher (en (37)) réfèrent à une action (ou absence d'action) dont la radicalité « tranche », justement, avec ce qui précède. Quoi qu'il en soit pour ces exemples, le mouvement argumentatif qui nous semble l'emporter est, plus proprement qu'un mouvement d'opposition, celui de l'aboutissement graduel d'un processus : processus par exemple, en (31), d'affaiblissement de l'usage politique d'un mot, dont l'étape ultime est la disparition pure et simple; ou encore, en (36), de remise à plus tard de l'action, qui finit en une totale inaction (les divers autres exemples suivent un schéma similaire).

Dans tous les cas ici, on retrouve un type d'effet de sens comparable à ceux des exemples de la section précédente, mais engendré par une accumulation des situations plutôt que par une simple opposition ou gradation d'intensité entre deux situations. C'est cet effet de sens que nous désignons comme « clôture » : effet de clôture qu'on pourrait gloser aussi bien par les images du «point» (culminant, final), ou de la " chute », et qui correspond à une mise en relief du dernier élément d'une série. Dans une configuration de fin d'énumération, aussi bien que dans les exemples de la section précédente, le rôle de $A V$ se rapproche assez de celui d'un adverbe conclusif tel que finalement (d'ailleurs effectivement présent en (36) : cf. avant, finalement, de ne rien faire $)^{16}$.

Précisons enfin que l'effet de clôture n'est pas lié systématiquement à un effet de renversement/ intensification de situation ; qu'il nous semble pouvoir en être détaché, comme en (39) ou (40) :

(39) [A la tribune, Martine Aubry se dandine sur sa chaise $]_{\mathrm{P} 1}$ et [lâche un «ouh!» dans un sourire... $]_{\mathrm{P} 2}$ avant $\mathrm{d}$ '[attribuer, sans répondre, le micro à une autre résidente. $]_{\mathrm{P} 3}$ (Libération, 23 juin 2011) ${ }^{17}$

(40) [On examine d'abord la terminologie et les lignes de partage au sein du champ linguistique (section 3). $]_{\mathrm{P} 1}$ [On prend ensuite en compte des éléments volontairement laissés de côté par Chevalier et Encrevé : la continuité de la recherche en philologie classique (section 4), l'influence du structuralisme au-delà de la linguistique, l'intérêt pour de nouvelles problématiques en linguistique (section 5). $]_{\mathrm{P} 2}$ [On essaiera aussi de voir l'influence du renouveau linguistique de la période 1958 - 1968 sur la période plus récente (section 6), ] $\mathrm{P}_{3}$ avant d'[en venir à la conclusion (section 7). $]_{\mathrm{P} 4}$ (T. Poibeau, Quelques remarques sur le " champ linguistique » en France dans l'après guerre (1950-1970))

Le cas d'un exemple tel que (39) n'est cependant pour nous pas tout à fait clair, dans la mesure où nous ne sommes pas sûre que, dans l'interprétation de la succession des événements (se dandiner sur sa chaise / lâcher un «ouh!» dans un sourire / attribuer, sans répondre, le micro à une autre résidente), il n'y a pas une tendance à opposer les deux premiers (réactions d'une personne flattée) au dernier (refus de s'attarder à la flatterie). C'est-à-dire qu'il nous semble que la présence d'une énumération tend à infléchir une lecture où $\mathrm{S}_{\mathrm{n}+1}$ est interprétée en relation d'opposition (ou de gradation) avec les situations qui la précèdent $^{18}$. Mais pour l'exemple (40) (relevé dans les actes de CMLF 2008), la possibilité d'existence indépendante de l'effet de clôture paraît plus assurée: dans ce type d'exemple en effet, où il s'agit d'annoncer un plan, le rôle du $A V$ peut se limiter plus facilement à introduire la conclusion (ainsi mise en relief par sa simple fonction de conclusion).

\subsection{Au final : des effets de mise en relief}

À ce point de la réflexion il apparaît que c'est bien la mise en relief de la situation introduite qui, en accord avec les propriétés de la subordination inverse telles que décrites par Combette 2011 (cf. notre introduction), est l'effet de sens majeur de $A V$ assertif. Cette mise en relief se décline sous des formes 
différentes (retournement/intensification de situation, ou clôture) suivant le type de configuration (présence d'une relation de contraste, ou d'une énumération) qui en favorise l'émergence. Ces formes ne sont pas exclusives les unes des autres, elles peuvent se combiner, aussi bien qu'exister indépendamment. Mais ce qui importe c'est que dans tous les cas, la mise en relief concerne le dernier élément. Dans tous les cas également, la mise en relief coöncide avec la résolution d'une forme d'attente, de suspens l'accumulation de situations $S_{1}-S_{n}$, pour le cas de l'énumération, s'avérant assez proche de la prolongation de durée de $\mathrm{S}_{1}$ évoquée pour les cas de contraste.

Nous faisons l'hypothèse que cet effet de suspens, pour $A V$ assertif aussi bien que pour quand inverse, tient aux particularités syntaxiques et pragmatiques de ces constructions, et plus précisément à ce que Béguelin (2005) désigne comme " dégroupage », lequel «affecte une clause qui se scinde en deux par une sorte de scissiparité » (p. 143). Ce «dégroupage» vaut selon Béguelin pour «certaines subordonnées, qui finissent par échapper à la sphère de la rection verbale pour s'émanciper syntaxiquement autant que pragmatiquement ». Le cas du quand inverse est en particulier ainsi mentionné, résultat de l'évolution de la séquence quand $+p$ régie «vers le statut d'énonciation autonome » (p. 143). C'est ce même dégroupage qui s'applique, selon nous, à la construction en $A V$ assertif $^{19}$; et c'est encore ce dégroupage qui selon nous, parce qu'il garde trace du « groupage » originel, favorise l'expression du suspens par une sorte d'équilibre instable entre autonomie d'une part, et dépendance de l'autre, de la proposition introduite par $A V$. En tâchant de le dire plus clairement : si $\mathrm{P}_{2}$ (ou $\mathrm{P}_{\mathrm{n}+1}$ ), la proposition introduite par $A V$, est comme nous l'avons dit autonome, elle ne l'est cependant pas au même titre qu'une proposition introduite par un connecteur tel que puis, ensuite ou mais. L'usage de la «conjonction» continue en effet d'impliquer une forme d'interdépendance entre $\mathrm{P}_{1}$ et $\mathrm{P}_{2}$, ou plus généralement (pour rendre compte des configurations de contraste aussi bien que d'énumération), entre $P_{n}$ et $P_{n+1}: P_{n} n$ 'est qu'en apparence achevée en l'absence de $P_{n+1}$ et $P_{n+1}$ ne peut exister sans $P_{n}$. Cette interdépendance permettrait de comprendre, croyons-nous, qu'au moment de l'énonciation de $P_{n+1}$, un effet de résolution de suspens puisse être engendré. Notre hypothèse resterait à étayer davantage sur le plan théorique ; nous la laisserons cependant ici en l'état.

Pour continuer de mettre en évidence la proximité des effets de sens associés aux diverses configurations, on mentionnera encore les quelques exemples suivants - lesquels permettront également de ne pas laisser penser que le phénomène que nous étudions est circonscrit au domaine de la presse. Car si elle est particulièrement prégnante dans la presse, la construction avec $A V$ assertif se retrouve également dans des œuvres littéraires récentes; elle nous est même apparue comme une construction de prédilection chez des auteurs tels que Thierry Jonquet, Jean Echenoz, et surtout Patrick Lapeyre (à qui les exemples cités sont empruntés) :

(41) [...] il s'échine pendant une partie de l'après-midi à traduire un article de revue médicale consacré au traitement de l'infibulation en Afrique, avant de jeter l'éponge et d'aller se chercher une bouteille de bière dans la cuisine. (Patrick Lapeyre, La vie est brève et le désir sans fin)

(42) Pendant quelques dixièmes de seconde, Blériot, qui est devenu très pâle [...], le regarde les yeux écarquillés, avant de réaliser qu'il n'a d'autre ressource que de lui tendre la main. (Ibid.)

(43) Il ne s'en rase pas moins méticuleusement, passe une lotion hydratante sur ses joues, avant de se masser longuement les tempes. (Ibid.)

(44) Il passe une partie de la journée ans un état d'anxiété flottante, la tête plongée dans son dictionnaire [...] puis tape une dizaine de lignes de sa traduction sur les lymphatiques, avant de rappeler enfin son père. (Ibid.)

(41) et (42) d'un côté, (43) et (44) de l'autre, illustrent des cas qu'on a déjà examinés de retournement de situation pour les premiers, de clôture d'énumération pour les seconds. Mais ce qui nous frappe plus particulièrement dans les nombreuses occurrences de avant de assertif relevées dans le roman de Patrick Lapeyre $^{20}$, et dont les quatre exemples ci-dessus sont parfaitement représentatifs, c'est la régularité avec laquelle elles se répartissent, pour la plupart, dans l'une ou l'autre de ces deux catégories. Nous parait remarquable également, telle qu'elle s'incarne dans ces exemples, la continuité de fonctionnement de la construction, qu'elle relève de l'une ou l'autre catégorie : dans tous les cas, c'est bien l'effet «dernier élément », mis en relief comme tel, qui l'emporte. 
Notons que ce «dernier élément » peut lui-même se subdiviser en plusieurs sous-éléments : c'est le cas par exemple en (41), où avant de (cf. avant de... et de...) étend sa portée à la fois sur jeter l'éponge et sur aller se chercher une bouteille de bière dans la cuisine. C'est le cas également, et de façon particulièrement flagrante, dans l'exemple (45) qui suit, où avant de (cf. avant de ... et de... puis de... puis de...) étend sa portée sur toute la succession des situations réapparaître / s'immobiliser / repartir / s'arrêter à nouveau :

(45) Sa silhouette colossale et légère disparaît par instants, interceptée par l'ombre des arbres, avant de réapparaitre à la lumière et de s'immobiliser en même temps que lui, puis de repartir, en s'imposant des mouvements parfaitement contrôlés et silencieux, puis de s'arrêter à nouveau.

Cette nouvelle succession de situations à la suite du avant de a cependant pour conséquence de «brouiller » l'effet de mise en relief qui lui est associé. En (41), on peut encore considérer que c'est la conjonction des situations jeter l'éponge et aller se chercher une bouteille de bière dans la cuisine qui, en opposition avec la situation s'échiner à traduire un article de revue médicale, fait l'objet d'un effet de retournement de situation. En (45), c'est beaucoup moins évident: s'il y a une opposition sémantique claire entre disparaître et réapparaître, favorable là encore à un effet de retournement de situation, cet effet semble pouvoir difficilement s'étendre à toutes les situations qui suivent (elles-mêmes en relation d'opposition les unes par rapport aux autres). Ce phénomène d'enchâssement de plusieurs propositions sous le $A V$ serait à examiner plus en détail. Nous n'irons, pour notre part, pas plus loin sur cette question, nous contentant de remarquer que ces propositions enchâssées paraissent elles-mêmes prendre une certaine autonomie énonciative $^{21}$; de remarquer, également, que dans la succession de $A V$ et de puis, associés à des configurations similaires de contraste, la différence entre les deux types de connecteurs semble s'amoindrir (l'association d'un effet de retournement de situation au seul $A V$ semblant alors quelque peu artificielle). C'est maintenant, précisément, à des cas où le fonctionnement de $A V$ paraît se rapprocher de celui d'un puis que nous allons nous intéresser.

\section{Présence / absence des effets de sens}

Revenons sur la question de la non-systématicité des effets de sens associés à $A V$ assertif. Pour les besoins de notre analyse nous nous sommes appliquée à rassembler des exemples représentatifs de tels effets de sens - des exemples, donc, qu'on peut regarder comme «bien choisis ». Or, au fur et à mesure de l'analyse, il nous est apparu que la grande majorité, sinon la totalité des exemples de $A V$ assertif que nous avons pu relever se conforment, en fait, à l'une ou l'autre des configurations présentées ci-dessus. Pour un relevé d'exemples fait « à la main », nous ne chercherons pas à présenter d'analyse quantitative ou statistique. Nous insisterons simplement sur le fait que, parmi les exemples que nous regardions initialement comme «neutres », i.e. dénués d'effets de sens, la plupart se sont avérés présenter des caractéristiques communes avec les exemples déroulés jusqu'ici. Ainsi nous avions observé, dans la presse, un emploi récurrent de $A V$ pour relier deux moments d'un dire, rapporté plus ou moins directement ou indirectement (cf. exemples (46) à (48)), ou d'un parcours biographique ${ }^{22}$ (cf. exemples (49) à (51)) :

(46) Objet politique non identifié, ce provocateur bon teint est capable de comparer Barack Obama à Hitler puis de confier qu'il mettrait bien "la petite moustache à Sarkozy», avant d'affirmer que l'influence des jeux vidéo violents ne peut être écartée dans le drame de Toulouse. (Libération, 6 avril 2012)

(47) «Après la mort de Franco, plus d'un million de personnes se rassemblait ici et peu à peu le nombre a diminué», admet José Luis Corral avant d'affirmer que leur rassemblement reste toutefois aujourd'hui «un acte représentatif». (Libération.fr, 24 novembre 2013)

(48) «Vous dites n'importe quoi», «vous ne connaissez pas le sujet!» a lancé le patron du Nouveau Centre, avant d'assurer, un peu vite, qu'il n'avait jamais rencontré l'émir de Dubaï, le cheikh Mohammed al-Maktoum. (Libération, 29 novembre 2011)

(49) Libéré en 1981, il entreprend une formation de coiffeur, puis d'électricien, avant d'exercer divers petits boulots de chauffeur et de manutentionnaire. En 1983, il replonge, pour le vol à main armée d'une voiture, [...] (Libération, 19 mai 2008) 
(50) Elle [Rebekah Brooks] prend en 2003 la direction du quotidien The Sun avant, en septembre 2009, de devenir directrice générale de News International, qui, outre le Sun et feu News of the World, compte aussi The Times et The Sunday Times. (Libération, 15 juillet 2011)

(51) Et la "stratégie Ovaldé » fut une tactique payante : elle est devenue, il y a quinze ans, fabricante pour les éditions du Seuil, avant d'intégrer, il y a deux ans, le secteur Jeunesse des éditions Albin Michel. (Télérama, 18 mars 2008)

Dans ce type d'exemples le $A V$ nous semblait, a priori, participer simplement à la progression narrative. Or, à y regarder de plus près, on y retrouve le plus souvent une configuration soit de contraste, soit d'énumération : nous laissons notre lecteur le vérifier aisément ci-dessus pour les exemples (46), (47) et (49). Restent des exemples tels que (48), (50) et (51), où il paraît effectivement impossible de voir dans le $A V$ autre chose qu'un marqueur de succession temporelle, proche d'un marqueur comme puis. C'est l'existence de tels exemples «neutres » que nous allons continuer d'explorer, à travers les deux exemples suivants :

(52) Dans ce qui suit nous allons rappeler quelques repères historiques (1.), préciser la démarche relative aux corpus (2.) et à leur analyse (3.), avant d'expliciter la manière dont s'imbriquent analyse de l'organisation de l'interaction et analyse des ressources linguistiques (4.), avant de conclure sur quelques enjeux majeurs qui ouvrent sur un chantier encore largement à défricher (5.) (L. Mondada, Contributions de la linguistique interactionnelle)

(53) De mai 1950 à 1955, elle [la prison de Sighet] ne désemplira pas, comptant, en moyenne, deux cents détenus, avant de recevoir de nouveau des droits communs, d'être occupée par les soldats soviétiques, de se reconvertir en usine à balais puis en dépôt de sel, avant de tomber en ruine. Une prison ? Plutôt un centre d'extermination lente. (Télérama, 29 novembre 2006)

Dans les deux cas, on a une succession assez étonnante de deux $A V$ assertifs - assez étonnante au sens où elle ne nous semble pas relever d'un usage tout à fait $\langle\text { classique }\rangle^{23}$. Aussi rare soit-elle, cette succession n'en témoigne pas moins de la possibilité pour $A V$ d'introduire une proposition dont le contenu ne fait l'objet d'aucune mise en relief particulière. En (52) en effet aussi bien qu'en (53), seul le second avant de est représentatif d'un effet de clôture ${ }^{24}$. De cet effet de clôture le premier avant de est exclu; et il n'y a pas davantage d'effet de sens lié à une configuration de contraste.

À ce point de l'analyse, la question que nous nous posons est de déterminer si l'existence de tels cas, même relativement peu fréquents, où $A V$ assertif ne s'accompagne d'aucun effet de mise en relief, est un obstacle à l'intégration de la construction dans la classe des constructions inverses - précisément caractérisées, nous l'avons vu, par une opposition entre premier et second plans.

Une première réponse possible serait de dire que sont inclus dans la subordination inverse les cas correspondant aux configurations de la section 3, et que sont exclus les autres. C'est à peu près ce que propose Combettes (2011) à propos des subordonnées consécutives, qui, selon lui, sont « particulièrement apte[s], sous certaines conditions, à remplir le rôle textuel de la SI [subordination inverse] » (p. 88) : ces conditions étant une répartition des formes verbales entre aspects sécant et global (avec « la présence d'un imparfait ou d'un plus-que-parfait de second plan dans la proposition régissante et l'emploi d'un passé simple dans la subordonnée » (p. 89)). Dans le cas d'emploi de deux formes d'aspect global, il n'y a, selon Combettes, qu'une "succession de premiers plans » (p. 89) et donc pas de subordination inverse. Combettes précise que le problème serait comparable pour les relatives narratives ou continuatives, avec une analyse dépendant là encore de la répartion des formes verbales. C'est-à-dire que les conditions de la subordination inverse sont, pour ces types de cas, clairement circonscrites. Pour notre cas du $A V$ assertif, elles resteraient plus difficiles à délimiter, car liées à des effets de sens moins strictement définis.

Une seconde réponse nous est inspirée par Maurel (1992), selon qui subordonnées consécutives aussi bien que relatives continuatives sont à rattacher au phénomène de subordination inverse, quelles que soient les formes verbales utilisées. C'était ce qui apparaissait déjà au début de notre introduction, avec les exemples (3) et (4) cités par Maurel (1992) en illustrations de constructions inverses. Reprenons ici l'exemple (3), avec une relative continuative :

(3) Il ouvrit la porte, qu'il referma aussitôt 
Pour cet exemple, l'analyse proposée est que «la relative marque une étape décisive dans la stratégie narrative du locuteur : c'est donc la "'principale"' » (p. 79).

Cette idée d' "étape décisive dans la stratégie narrative du locuteur » (à mettre en relation avec l'effet de « mise en relief » mentionné par Combettes (2011)) s'avère, pour nous, particulièrement pertinente et utile; nous la retiendrons dans la mesure où elle nous permet de rendre compte du cas qui nous occupe où, justement, la distinction des premier et second plans ne dépend pas de l'emploi des formes verbales. Ce faisant, nous perdons de la rigueur de définition des conditions de la subordination inverse, telles qu'elles sont décrites par Combettes (2011). Mais nous pouvons ainsi proposer un traitement plus homogène du $A V$ assertif. Certes «subjective », l'idée d'étape décisive dans la narration n'en est pas moins révélatrice. Elle permet d'apporter un éclairage nouveau sur l'ensemble de nos exemples, où les cas neutres apparaîtraient simplement comme une sorte de " cas limites », une forme minimale à partir de laquelle, dans des contextes le plus souvent adéquats, des effets de sens plus marqués sont susceptibles d'émerger. Nous finirons sur ce point en mentionnant deux exemples encore, d'autant plus précieux pour notre analyse qu'ils font coexister construction avec $A V$ assertif et relative continuative :

(54) L'individu a été interpellé peu de temps après les faits, qu'il a vite reconnu [sic], avant d'être écroué et mis en examen pour viol aggravé (sous la menace d'une arme) et tentative d'assassinat. (metronews.fr, 18 février 2011)

(55) Les travaux de l'expert en sécurité n'ont pas tardé à faire le tour de quelques blogs d'actualité technologique, avant de remonter aux oreilles de Carrier IQ... qui n'a pas apprécié la mauvaise publicité. (liberation.fr, 30 novembre 2011) $)^{25}$

Que la relative soit placée avant (cf. (54)) ou après (cf. (55)) la contruction en $A V$ importe peu : dans les deux cas, chacune des propositions constitue au moment de son énonciation (donc, chacune à son tour) une étape décisive par rapport à la proposition qui précède : reconnaître les faits marque dans un premier temps une étape décisive par rapport à être interpellé, puis dans un second temps, être écroué et mis en examen par rapport à reconnaître les faits (en (54)); remonter aux oreilles de Carrier IQ marque une étape décisive par rapport à ne pas tarder à faire le tour de quelques blogs, puis ne pas apprécier la mauvaise publicité par rapport à remonter aux oreilles de Carrier IQ (en (55)).

\section{Conclusion}

Notre conclusion sera brève. Nous nous contenterons de souligner la proximité entre $A V$ assertif et quand inverse, beaucoup plus grande qu'il n'y paraissait de prime abord. De préciser également que cette occultation préalable des points de convergence peut, sans doute, trouver une explication dans le fait que les effets de sens associés à $A V$ dépendent davantage et plus diversement du contexte, ne pouvant s'appuyer sur un marquage aussi clair et systématique que celui de l'aspect verbal, dans le cas de quand inverse. Là encore cependant, il faudrait nuancer, puisque l'opposition entre aspect sécant et global ne suffit pas non plus à assurer l'interprétation inverse de quand, et que d'autres conditions sont requises (explorées dans le détail par Vogeleer, 1998).

En perspective, enfin, nous insisterons sur le fait que le $A V$ assertif, à la différence du quand inverse, relève d'un usage que nous qualifions faute de mieux d' " assez récent » (cf. note 19) : un usage donc qui mériterait, pour une meilleure compréhension du phénomène, de faire l'objet d'une étude diachronique.

\section{Références bibliographiques}

Asher, N. (1993). Reference to Abstract Objects in Discourse. Dordrecht : Kluwer.

Béguelin, M.-J. (2005). Coalescences et dégroupages syntaxiques : réflexion sur les conditions pragmatiques et sémantiques des grammaticalisations. In Auchlin, A. et alii (éds), Structures et discours. Mélanges offerts à E. Roulet, Québec : Les Editions Nota Bene, 139-150.

Benzitoun, C. (2006). Description morphosyntaxique du mot quand en français contemporain. Thèse de doctorat: Université d'Aix-Marseille 1. 
Benzitoun (2013). Faut-il remettre les pendules de la subordination temporelle à l'heure ? Description de deux fonctionnements de quand et avant que / de. Cahiers Chronos, 26, 419-435.

Borillo, A. (1988). Quelques remarques sur quand connecteur temporel. Langue Française, 77, 71-91.

Chétrit, J. (1971). Syntaxe de la phrase complexe à subordonnée temporelle. Paris : Klincksieck.

Combettes, B. (2011). Subordination inverse et opposition des plans à l'époque classique. In Corminboeuf, G. \& Marie-José Béguelin, M.-J. (éds), Du système linguistique aux actions langagières. Mélanges en l'honneur d'Alain Berrendonner, Bruxelles : De Boeck-Duculot, 83-94.

Corminboeuf, G. (2007). Coordination, subordination, corrélation ou énonciation autonome ? Une analyse syntaxique des constructions du type Que je bouge (et) il me ramènera vite à l'ordre. Travaux neuchâtelois de linguistique, $47,177-194$

Declerck, R. (1997). When-clauses and temporal structure. London : Routledge.

Deulofeu, H.-J. (1988). Syntaxe de que en français et le problème de la subordination. Recherches sur le français parlé, $8,79-104$.

Ducrot, O. (1980). Analyses pragmatiques. Communications, 32, 11-60.

Ducrot, O. (1991). Dire et ne pas dire. Paris : Hermann (troisième édition corrigée et augmentée).

Hamann, C. (1989). English temporal Clauses in a Reference Frame Model. In Schopf, A. (ed.), Essays on Tensing in English, Vol. II : Time, Text and Modality, Tübingen : Niemeyer, 31-154.

Hansen, M.-B. M. (2005). A comparative study of the semantics and pragmatics of enfin and finalement, in synchrony and diachrony. Journal of French Language Studies, 15/2, 153-171

Lambrecht, K. (1994). Information structure and sentence form. Cambridge : Cambridge University Press.

Le Draoulec, A. (2005). Avant que / de : possibles passages à la connexion temporelle. Journal of French Language Studies, 15, 131-151.

Le Draoulec, A. (2006). Avant que- or Avant de-Clauses: When Presupposition Gives Way to an 'Assertive Construction'. in Ch. Nishida \& J.-P. Montreuil (eds), New Perspectives on Romance Linguistics, vol. I : Morphology, Syntax, Semantics, and Pragmatics, Amsterdam/Philadelphia, John Benjamins Publishing Company, 155-168.

Le Draoulec, A. (2008). «Il retombe puis rebondit avant de retomber à nouveau... » : puis et avant que dans la progression narrative. In Birkelund, M. ; Mosegaard Hansen, M.-B. M. \& Norén, C. (éds), L'énonciation dans tous ses états, Mélanges offerts à Henning Nølke, Berne : Peter Lang, 403-432.

Maurel, J.-P. (1992). Subordination inverse et neutralisation du relatif. Travaux linguistiques du Cerlico, 5, 72-88.

Olsson, L. (1971). Étude sur l'emploi des temps dans les propositions introduites par quand et lorsque et les propositions qui les complètent en français contemporain. Uppsala : Acta Universitatis Upsaliensis.

Sechehaye, A. (1926). Essai sur la structure logique de la phrase. Paris : Champion.

Shelling, M. (1982). Quelques modalités de clôture : les conclusifs finalement, en somme, au fond, de toute façon. Cahiers de linguistique française, 4, 63-106.

Vogeleer, S. (1998). Quand inverse. Revue québécoise de linguistique, 26/1, 79-101.

Wilmet, M. (2010). « À peine avions-nous poussé un cri de surprise qu'il en arriva une seconde » : considérations sur la subordination inverse. In Béguelin, M.-J.; Avanzi, M. \& Corminboeuf, G. (éds), La Parataxe Tome 1, Entre dépendance et intégration, Berne : Peter Lang, 69-90.

\footnotetext{
${ }^{1}$ On utilisera ce type de raccourci commun pour désigner une construction de subordination inverse introduite par quand (ou lorsque).

${ }^{2}$ Pour l'équivalent anglais when.

${ }^{3}$ Les constructions en à peine (ou pas plus tôt/sitôt)... que sont communément regardées comme relevant de la subordination inverse. Pour les constructions suivantes, il s'agit plutôt d'un élargissement de la notion, qui ne fait pas
} 
l'objet d'un consensus : nous nous contentons ici de renvoyer aux arguments - non convergents - développés par Maurel (1992) et Combettes (2011) (et que nous reprendrons partiellement en section 4).

${ }^{4}$ Le quand inverse étant lui-même parfois qualifié de quand «narratif » (cf. Declerck, 1997).

5 Parmi les divers termes génériques couramment utilisés pour recouvrir événements et états («situation», « procès », « éventualité »), nous retenons le premier.

${ }^{6}$ Nous soulignons en gras dans nos exemples, en plus des avant de / avant que, les éventuels éléments sur lesquels nous souhaitons attirer l'attention.

${ }^{7}$ Et qui correspond, pour le $A V$ assertif, à une contrainte de continuité thématique que nous ne détaillons pas ici (cf. Le Draoulec, 2008).

${ }^{8}$ Le fait que la grande majorité des exemples de contruction assertive soient des exemples avec avant de plutôt que avant que pourrait nous encourager à ne retenir que le avant de : c'est cependant pour ne pas exlure la possibilité d'exemples du type de (14) que nous tenons à préserver, dans des descriptions générales, la mention des deux formes (à travers notre $A V$ ).

${ }^{9}$ Cette possibilité d'enchaînement est, selon nous, le meilleur argument en faveur de l'hypothèse selon laquelle la construction étudiée est bien une construction assertive : une construction dont on ne pourrait "récupérer » le caractère présuppositionnel à l'aide du concept d' «accommodation conventionnalisée » ou " grammaticalisée » tel qu'appliqué par Lambrecht (1994 : 70-71) à d'autres cas de figure (en particulier, des cas de construction clivée).

${ }^{10}$ Notons que dans cet exemple, le second avant de (avant d'être placé face à cette plainte civile) n'est pas assertif mais présuppositionnel (il est antéposable, et ne donnerait pas lieu à enchaînement).

${ }^{11}$ En l'absence d'enchaînement effectif, la reconnaissance d'une possibilité d'enchaînement repose sur un jugement linguistique qui n'est pas toujours très sûr: d'où les cas d'indétermination, évoqués par Le Draoulec (2005), susceptibles de se prêter aussi bien à l'une ou à l'autre analyse (présuppositionnelle ou assertive).

12 Dans un cadre formel tel celui de la Segmentend Discourse Representation Theory (SDRT) (initialement développée par Asher, 1993), la relation de Contraste entre deux constituants est déclenchée par un isomorphisme partiel des arbres syntaxiques de ces constituants (isomorphisme que suffit à assurer l'identité de sujet syntaxique), associé à la présence de thèmes contrastifs.

${ }^{13}$ Le terme de volte-face ici, s'il ne désigne à proprement parler qu'une éventuelle future volte-face, vaudrait tout aussi bien pour qualifier le passage de catégoriquement refuser l'invitation à se raviser.

${ }^{14}$ Il faudrait examiner plus précisément ce qu'il en serait en SDRT (sachant cependant que l'attribution des relations de discours n'y est pas toujours clairement tranchée). Quoi qu'il en soit, et ainsi que le signale l'un de nos relecteurs (que nous remercions ici), un problème se pose du fait qu' «il n'y a pas de contraste au plan argumentatif »: $\mathrm{P}_{1}$ et $\mathrm{P}_{2}$, en effet, ont la même orientation argumentative, " ce qui rend le terme de contraste relativement contre-intuitif ».

${ }^{15}$ Sans compter la difficulté plus proprement « technique » que nous avons à décider s'il convient d'inclure dans les propositions ainsi segmentées des éléments tels que connecteurs et adverbiaux cadratifs (cf. les nombreux puis, ou encore le Dans un premier temps qu'on a en (31)). Nous choisissons de ne pas les inclure, de la même façon que nous n'incluons pas les avant de / avant que (ce choix n'a de fait que peu d'importance ici, où il s'agit uniquement de donner une visualisation approximative des limites des propositions).

${ }^{16}$ Pour une exploration du rôle pragmatique de finalement, cf. Shelling (1982), Hansen (2005).

${ }^{17}$ L'exemple (39) apparait, dans l'article de Libération dont il extrait, dans le prolongement de l'exemple (30) cité plus haut.

${ }^{18}$ Alors qu'inversement, bien sûr, la présence d'une opposition ou gradation entre $\mathrm{S}_{1}$ et $\mathrm{S}_{2}$ (comme dans les exemples de la section 3.1.) n'invite pas à recréer à partir de $S_{1}$ un ensemble possible de situations énumérées, que $S_{2}$ viendrait clore.

${ }^{19}$ Supposant un phénomène de grammaticalisation (objet de l'étude de Béguelin) qu'il conviendrait d'explorer en diachronie, mais que nous passerons à peu près sous silence ici, nous contentant de noter notre intuition selon laquelle le développement de l'usage du $A V$ assertif est assez récent. 
${ }^{20}$ Nous comptons en tout, dans l'intégralité du roman (par comptage automatisé sur tablette de lecture), 78 occurrences de constructions en avant de (ou avant d') postposées (contre 5, seulement, qui ne le sont pas); et parmi ces 78 occurrences, 71 nous paraissent clairement assertives (vs 7 occurrences, donc, présuppositionnelles, du type de "Il lui reste trente minutes avant de rencontrer Nora »). Notons également la différence flagrante entre l'usage de avant de et de avant que : on ne trouve pas un seul avant que/qu'assertif dans tout le roman (absence correspondant à une quasi-absence plus générale des avant que/qu' tout court, dont on ne compte que 3 occurrences, et 1 seule introduisant une subordonnée postposée).

${ }^{21}$ Cf. encore l'exemple suivant, où l'autonomie énonciative de la proposition enchâssée est marquée par une ponctuation forte (un point) :

(i) Mariée très jeune à un lointain cousin, elle aurait eu une fille, avant de perdre son époux. Et de décider d'émigrer vers New York, il y a neuf ans, pour rejoindre sa sœur aînée qui avait déjà fait le voyage. (Libération, 7 juillet 2011)

${ }^{22}$ Où il apparaît le plus souvent en concomitance avec un jalonnement temporel donné par divers adverbiaux de temps. Notons que la concomitance peut aller jusqu'à une insertion à l'intérieur du $A V$ (cf. en (50) avant, en septembre 2009, de...) ; insertion qui, dans certains cas, conduit à des alliances étonnantes, comme dans l'exemple ci-dessous entre avant de et plus tard:

(ii) Il [Floyd Landis] a découvert le vélo à quinze ans et a très vite disputé des compétitions contre la volonté de ses parents. En 1993, il remporte les Championnats nationaux de VTT, avant, deux ans plus tard, de quitter sa famille pour s'installer en Californie, où il choisit de se tourner vers le cyclisme sur route. (L'Équipe, 28 juillet 2006).

${ }^{23}$ Sur le plan plus proprement syntaxique, on remarquera que l'analyse de la façon dont les deux avant de sont hiérarchisés l'un par rapport à l'autre ne va pas sans poser problème. En (52), le second avant de apparaît au même niveau que le premier (et en clôture de l'énumération rappeler / préciser / expliciter). En (53) en revanche, le second apparaît sous la portée du premier (en clôture cette fois de l'énumération interne recevoir de nouveau des droits communs / être occupée par les soldats soviétiques / se reconvertir en usine à balais / puis en dépôt de sel).

${ }^{24}$ En parallèle, pour (52), avec le avant de conclusif de l'exemple (40) (extrait des mêmes actes de CMLF 2008).

${ }^{25}$ Qu'il s'agisse bien, en (55), d'une relative continuative n'est pas tout à fait assuré : la présence de points de suspension avant la relative nous paraît cependant aller en ce sens. 